\title{
Intramedullary Spinal Cord Solitary Metastasis as the Inaugural Presentation of Colorectal Cancer: A Case Report
}

\author{
Mafalda Mendes-Pinto ${ }^{\mathrm{a}, \mathrm{d}}$, Joana Parra ${ }^{\mathrm{b}}$, Orlando Galego ${ }^{\mathrm{a}}$, Bruno Costa-Gomes ${ }^{\mathrm{a}}$, \\ Marta Costa ${ }^{\mathrm{c}}$, Ricardo Veiga ${ }^{\mathrm{a}}$, Helena Gens ${ }^{\mathrm{b}}$
}

\begin{abstract}
This is a case report of intramedullary spinal cord metastasis (ISCM) of a colorectal cancer (CRC). A 67-year-old male with no relevant medical history presented with paresthesias in the lower limbs and paraparesis for 7 days. Thoracic spinal cord magnetic resonance imaging (MRI) demonstrated an oval intramedullary lesion suggestive of metastatic lesion. Primary tumor was a rectosigmoid transition zone invasive adenocarcinoma. He had a rapidly progressive neurological condition and became paraplegic with urinary and fecal incontinence. A conservative approach was performed, with spinal cord radiotherapy and derivate colostomy. The patient died 3 months later. This case report describes ISCM from CRC. Although it represents a rare clinical entity, we must be alert in order to achieve the earliest possible diagnosis trying to preserve the patient's quality of life.
\end{abstract}

Keywords: Rectosigmoid transition zone; Spinal cord; Intramedullary lesion; Metastatic disease; Magnetic resonance imaging

\section{Introduction}

Intramedullary spinal cord metastasis (ISCM) occurs in only $0.1-0.4 \%$ of all cancer patients [1-4]. It is more often associated with primary lung cancer, especially small-cell carcinoma, or breast cancer [3]. Colorectal cancer (CRC) represents only $3-5 \%$ of cases [3], with very few cases reported in the literature so far $[2,5]$. ISCM may represent a diagnostic challenge,

Manuscript accepted for publication November 18, 2016

aNeuroradiology Department, Centro Hospitalar e Universitario de Coimbra, Coimbra, Portugal

bNeurology Department, Centro Hospitalar e Universitario de Coimbra, Coimbra, Portugal

${ }^{\mathrm{c}}$ General Surgery Department, Centro Hospitalar e Universitario de Coimbra, Coimbra, Portugal

${ }^{\mathrm{d} C}$ Corresponding Author: Mafalda Mendes-Pinto, Neuroradiology Department, Centro Hospitalar e Universitario de Coimbra, Praceta Prof. Mota Pinto, 3000075 Coimbra, Portugal. Email: mafaldamendespinto@hotmail.com

doi: https://doi.org/10.14740/jmc2697w particularly in the rare cases where it is the first manifestation of the malignancy. This occurred in $22.5-39 \%$ of ISCM cases $[3,6]$. However, the diagnosis tends to become more frequent and easier with the development of newer and better imaging methods, mainly spinal cord magnetic resonance imaging (MRI) [1-3, 5-12].

Yet, ISCM still has a very poor prognosis because at diagnosis patients usually have very advanced disease with widespread metastasis $[5,6,8,11,13]$, with median survival rates of 4 months since the first symptoms [12].

ISCM should thus be promptly considered in the differential diagnosis of every patient with new onset spinal cord symptoms.

\section{Case Report}

A 67-year-old Caucasian male with no relevant medical history was admitted to the emergency department with paresthesias in the lower limbs and paraparesis with progressive worsening over the last 7 days.

On initial medical examination, the patient presented mild wasting and had adequate color and hydration of skin and mucous membranes. Neurological examination showed mild paraparesis, lower limb hyporreflexia, absent superficial abdominal reflexes, hypoesthesia and hypopalesthesia bellow D12 level and inability to walk without bilateral assistance.

Blood analytical evaluation revealed mild normocytic normochromic anemia. CSF examination showed a slight increase in protein count without neoplastic cells. There were no significant changes in computed tomography (CT) scan of the brain and spine.

The patient was admitted to the neurology ward with the diagnosis of transverse myelopathy of undetermined etiology. Treatment was immediately initiated with high doses of steroids (intravenous methylprednisolone $1 \mathrm{~g}$ for 5 days); nevertheless, his neurological condition kept deteriorating and he became paraplegic in less than a week. He also developed lower limb neuropathic pain and urinary and fecal incontinence 3 days later.

Thoracic spinal cord MRI demonstrated an oval intramedullary lesion causing medullary mild expansion in the level of D12 associated with extensive hypersignal from the medul- 


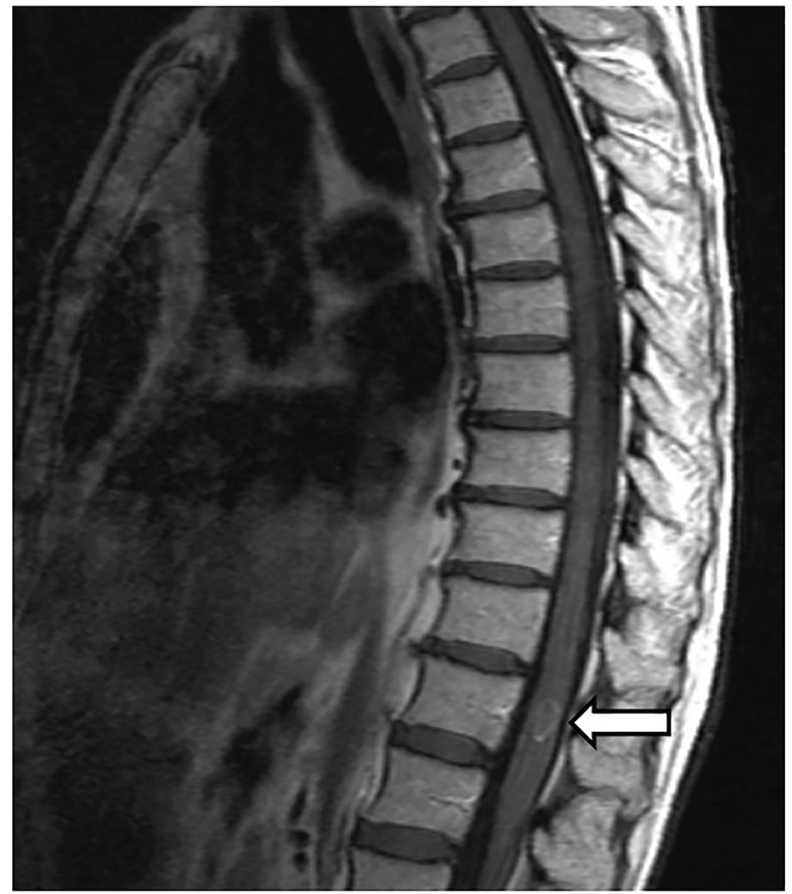

Figure 1. Sagittal view of magnetic resonance imaging with enhancement showing an intramedullary spinal cord lesion at D12 level (arrow)

lary cone to approximately D3 (Fig. 1). The features of this intramedullary expansive lesion were suggestive of metastatic lesion.

The search for a primary tumor was initiated. A thoracicabdominal-pelvic CT scan demonstrated the presence of a lesion with neoplastic characteristics in the rectosigmoid transition zone with various lymph nodes conglomerates and several subpleural nodules, suggesting metastatic lesions. Whole-body positron emission tomography with 18 fluorodeoxyglucose (PET-18 FDG) was also performed and confirmed the findings of the CT scan and also the presence of a focal hypermetabolic lesion at D12.

Rectosigmoidoscopy confirmed the presence of rectosigmoid transition zone adenocarcinoma with extraparietal invasion and several lymph nodes conglomerates staged preoperatively as T4aN2 (TNM staging system) in pelvic MRI (Fig. 2).

The case was discussed by a multidisciplinary team (Neurosurgery, Oncology, General Surgery, Radiology and Radiotherapy) concluding that there was no indication for neurosurgical intervention, given the advanced stage of the primary tumor with spread metastasis and the fact that the patient was paraplegic. The patient completed five spinal cord radiotherapy $(20 \mathrm{~Gy} / \mathrm{F} 5 / 1 \mathrm{~S})$ treatments associated with oral corticotherapy to relieve severe pain in the lower limbs. Later, the patient underwent laparotomy but the primary tumor was considered unresectable and only a derivative colostomy was performed. In a further multidisciplinary team meeting, adjuvant palliative chemotherapy was proposed but never carried out because of the patient's poor general condition.

The patient attended a convalescence home for 3 months and his clinical condition continued worsening with multiple

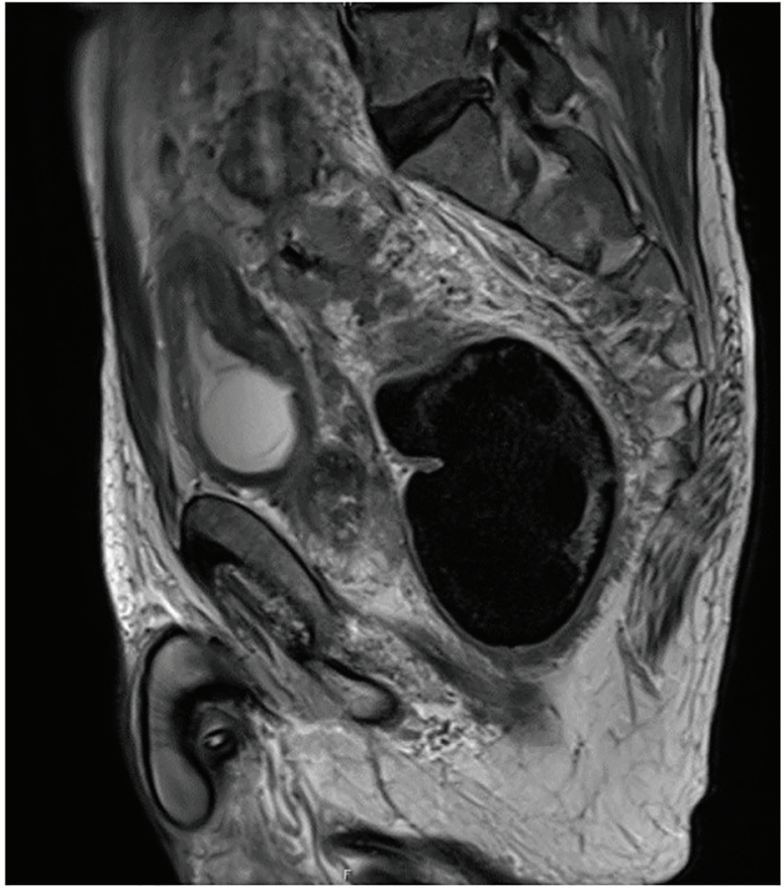

Figure 2. Sagittal view of pelvic magnetic resonance imaging revealing primary tumor at rectosigmoid transition zone.

infectious complications until he died 4 months after ISCM diagnosis.

\section{Discussion}

ISCM represents only $1-3 \%$ of spinal cord tumors [4] and CRC is one of the less frequent tumors associated with it. According to Sung et al [12], the median age at presentation of ISCM is 56 years (range: $4-82$ years) and it usually manifests by muscle weakness, back pain and radicular pain. Sensory loss and bowel and bladder dysfunction are typically seen at a later stage $[4$, 6], although there are cases (1\%) of asymptomatic patients [6].

ISCM as etiology of medullary lesion can be difficult to diagnose, especially in situations with no known primary tumor, as our case report. However, thanks to advances and wider use of imaging techniques, especially MRI, increase in the diagnosis of ISCM is happening [8]. As MRI is highly sensitive and many patients have a poor performance status to undergo a neurosurgical intervention, we can go without histological analysis and assume ISCM diagnosis only by imaging [2]. Our patient had no indication for neurosurgical intervention, even for biopsy, and the ISCM diagnosis was made by MRI confirmed by PET-18-FDG, which can be used to evaluate this clinical situation when MRI is unavailable [11].

The presence of ISCM usually appears associated with widespread metastasis $[5,7,12,13]$, including brain involvement in $61 \%$ of cases [12], with $80 \%$ mortality rates in first 3 - 4 months after the onset [11]. Our patient had already lung and pelvic but not brain metastasis at ISCM diagnosis.

As far as the treatment is concerned, there is no consen- 
sus because of the rarity of this entity $[2,12]$ and the lack of controlled studies [6]. The main objective is to try to preserve motor function in patients with rapid neurologic deterioration, considering conservative approach for patients with little or no cord function $[6,12]$. There are three main therapeutic tools: radiotherapy, chemotherapy and surgery. Radiotherapy with or without corticotherapy represents the gold standard to reduce edema in radiosensitive tumors [11]. The conservative treatment is often chosen because of advanced stage of disease and poor clinical condition of patients. On the other hand, we can consider surgery in certain patients, accordingly to their age, physical condition, location and severity of the primary tumor, other metastasis and surgical risk [4] and may offer some improvement in neurologic condition and quality of life in some patients $[4,5,10,12]$.

Despite an early diagnosis, our patient had already advanced disease, with disseminated metastasis and irreversible neurological deficits and so a very poor prognosis. He was in no condition to undergo neurosurgical intervention. Thus, only palliative approach to control his pain was performed.

\section{Conclusion}

ISCM from CRC is a rare condition, but thanks to therapies' improvement, cancer patients are living longer. This, associated with more advanced imaging methods, explains the fact that ISCM is being increasingly diagnosed. It is of uttermost importance to consider this diagnosis even in patients whose primary tumor is not usually associated with ISCM, such as in CRC, or even in undiagnosed primary tumors, as demonstrated by our case report. The management of these patients needs a multidisciplinary approach, requiring early spinal cord MRI and whole-body imaging for tumoral staging, in order to achieve timely diagnosis and treatment. The aim is to avoid irreversible neurologic deficits, preserving patients' quality of life.

\section{Grant Support}

None.

\section{Conflicts of Interest}

None.

\section{References}

1. Kaballo MA, Brennan DD, El Bassiouni M, Skehan SJ,
Gupta RK. Intramedullary spinal cord metastasis from colonic carcinoma presenting as Brown-Sequard syndrome: a case report. J Med Case Rep. 2011;5:342.

2. Kyung Ho Yang, Hye Ran Lee, Seong Yoon Yi, Joo Hyuk Jung, Seung Hee Kang, Pyong Hwa Choi. Intramedullar Spinal Cord Metastasis From Rectal Cancer. Annals of Coloproctology. 2014;30(5):237-240.

3. Duransoy YK, Mete M, Selcuki M, Isisag A. Isolated solitary intramedullary spinal cord metastasis presenting as the first manifestation of small-cell lung cancer: report of a rare case. Case Rep Neurol Med. 2012;2012:617280.

4. Pierpaolo Lunardi, Rodolfo Corinaldesi, Tommaso Lunardi, Roberto Floris. Solitary Intramedullary Spinal Cord Metastasis from Colon Carcinoma: A Case Report and Literature Review. Open Journal of Modern Neurosurgery. 2014;4:47-51.

5. Tai-Hsin Tsai, I-Cheng Lin, Pei-Chen Lin, Chieh-Hsin $\mathrm{Wu}$, Chih-Lung Lin, Yu-Feng Su. Intramedullary spinal cord metastasis from colon cancer: analysis of 19 reported cases. Spinal Cord Series and Cases. 2016;2:15026.

6. Ondrej Kalita. Current Insights into Surgery for Intramedullary Spinal Cord Metastases: A Literature Review. International Journal of Surgical Oncology. 2011. Article ID 989506.

7. Marc D. Winkelman. Intramedullary spinal cord metastatic tumors. Journal of Neurosurgery. 1985;62(2).

8. Rykken JB, Diehn FE, Hunt CH, Schwartz KM, Eckel LJ, Wood CP, Kaufmann TJ, et al. Intramedullary spinal cord metastases: MRI and relevant clinical features from a 13year institutional case series. AJNR Am J Neuroradiol. 2013;34(10):2043-2049.

9. Khan MN, Sharfuzzaman A, Mostafa MG. Spinal cord compression as initial presentation of metastatic occult follicular thyroid carcinoma. J Neurosci Rural Pract. 2014;5(2):155-159.

10. Ogino M, Ueda R, Nakatsukasa M, Murase I. Successful removal of solitary intramedullary spinal cord metastasis from colon cancer. Clin Neurol Neurosurg. 2002;104(2):152-156.

11. Hrabalek L. Intramedullary spinal cord metastases: review of the literature. Biomed Pap Med Fac Univ Palacky Olomouc Czech Repub. 2010;154(2):117-122.

12. Sung WS, Sung MJ, Chan JH, Manion B, Song J, Dubey A, Erasmus A, et al. Intramedullary spinal cord metastases: a 20-year institutional experience with a comprehensive literature review. World Neurosurg. 2013;79(34):576-584.

13. Kaya RA, Dalkilic T, Ozer F, Aydin Y. Intramedullary spinal cord metastasis: a rare and devastating complication of cancer - two case reports. Neurol Med Chir (Tokyo). 2003;43(12):612-615. 